MATHEMATICS OF COMPUTATION

Volume 70, Number 234, Pages 579-593

S $0025-5718(00) 01239-4$

Article electronically published on March 3, 2000

\title{
SIMPLE FINITE ELEMENT METHOD \\ IN VORTICITY FORMULATION FOR INCOMPRESSIBLE FLOWS
}

\author{
JIAN-GUO LIU AND WEINAN E
}

\begin{abstract}
A very simple and efficient finite element method is introduced for two and three dimensional viscous incompressible flows using the vorticity formulation. This method relies on recasting the traditional finite element method in the spirit of the high order accurate finite difference methods introduced by the authors in another work. Optimal accuracy of arbitrary order can be achieved using standard finite element or spectral elements. The method is convectively stable and is particularly suited for moderate to high Reynolds number flows.
\end{abstract}

\section{INTRODUCTION}

In this paper, we present a very simple finite element method for the unsteady incompressible Navier-Stokes equations (NSE) in vorticity-vector potential formulation. Let us first present the idea for the $2 \mathrm{D}$ case, where the vector potential is reduced to a stream function. The NSE read

$$
\begin{aligned}
\partial_{t} \omega+(\boldsymbol{u} \cdot \nabla) \omega & =\nu \Delta \omega, \\
\Delta \psi & =\omega,
\end{aligned}
$$

with no-slip boundary condition

$$
\psi=0, \quad \frac{\partial \psi}{\partial \boldsymbol{n}}=0,
$$

and the velocity is given by

$$
\boldsymbol{u}=\nabla^{\perp} \psi, \quad \nabla^{\perp}=\left(-\partial_{y}, \partial_{x}\right) .
$$

Adding inhomogeneous terms to the boundary condition only amounts to minor changes in what follows. For simplicity we will only consider a simply connected domain in this paper.

Finite difference approximations of (1.1)-(1.3) for large Reynolds numbers have been studied in detail in 9, 10, 11, where the the convection and viscous terms are both treated explicitly in the vorticity transport equation. The stream function, and hence the velocity, is then evaluated from the vorticity via the kinematic equation (the second equation in (1.1)). We developed a time-stepping procedure in which the value of the vorticity on the boundary can be obtained explicitly from the

Received by the editor June 8, 1999.

2000 Mathematics Subject Classification. Primary 65M60, 76M10.

Key words and phrases. Simple finite element method, Navier-Stokes equations, vorticity boundary conditions, error analysis.

JGL was supported in parts by NSF grant DMS-9805621.

(C)2000 American Mathematical Society 
stream function without any iteration, thus eliminating some traditional difficulties associated with the vorticity formulation [21]. The classical fourth order explicit Runge-Kutta time stepping method was used to overcome the cell Reynolds number constraint [9]. The resulting scheme is stable under the standard convective CFL condition. The method is very efficient. At each time step or Runge-Kutta stage, a standard Poisson equation is solved [9. In a fourth order compact difference scheme an additional mass-like matrix needs to be inverted [10.

Using a variational formulation and a finite element approximation gives a very general, natural, and clean way to implement the above methodology. It overcomes the difficulty of finding an accurate local vorticity formula for a curved boundary, especially in the $3 \mathrm{D}$ case. The NSE (1.1)-(1.3) can be formulated in a weak form 13]: find $\omega \in H^{1}(\Omega)$ and $\psi \in H_{0}^{1}(\Omega)$ such that

$$
\begin{aligned}
\left\langle\varphi, \partial_{t} \omega\right\rangle-\langle\nabla \varphi, \omega \boldsymbol{u}\rangle & =-\nu\langle\nabla \varphi, \nabla \omega\rangle, \quad \forall \varphi \in H_{0}^{1}, \\
\langle\nabla \varphi, \nabla \psi\rangle & =-\langle\varphi, \omega\rangle, \quad \forall \varphi \in H^{1} .
\end{aligned}
$$

The velocity is again given by (1.3). Here $\langle$,$\rangle is the notation for the standard inner$ product between functions on $\Omega$ and $\|\cdot\|$ for the $L^{2}$ norm.

In this form the first equation is taken as the evolution equation that updates $\omega$, and the second equation is then used to find $\psi$. However we can also put (1.4) in a more symmetric form: find $\psi \in H_{0}^{1}(\Omega)$ and $\omega \in H^{1}(\Omega)$ such that

$$
\begin{array}{rlr}
\left\langle\nabla \varphi, \nabla \partial_{t} \psi\right\rangle+\langle\nabla \varphi, \omega \boldsymbol{u}\rangle & =\nu\langle\nabla \varphi, \nabla \omega\rangle, \quad \forall \varphi \in H_{0}^{1}, \\
\langle\varphi, \omega\rangle & =-\langle\nabla \varphi, \nabla \psi\rangle, \quad \forall \varphi \in H^{1} .
\end{array}
$$

In this form the first equation is viewed as an evolution equation for updating $\psi$ and the second equation is used as a definition of $\omega$.

Let $X_{h}^{k}$ be the standard continuous finite element space [6] with $k$ th degree polynomials on each element of a triangulation $\mathcal{T}_{h}=\{K\}$. Denote by $h$ the maximum size (diameter) of the elements. Let $X_{0, h}^{k}$ be the subspace of $X_{h}^{k}$ with zero boundary values. The finite element approximation, treating time as continuous for now, is given as follows. Find $\omega_{h} \in X_{h}^{k}$ and $\psi_{h} \in X_{0, h}^{k}$ such that

$$
\begin{aligned}
\left\langle\varphi, \partial_{t} \omega_{h}\right\rangle-\left\langle\nabla \varphi, \omega_{h} \boldsymbol{u}_{h}\right\rangle & =-\nu\left\langle\nabla \varphi, \nabla \omega_{h}\right\rangle, \quad \forall \varphi \in X_{0, h}^{k}, \\
\left\langle\nabla \varphi, \nabla \psi_{h}\right\rangle & =-\left\langle\varphi, \omega_{h}\right\rangle, \quad \forall \varphi \in X_{h}^{k},
\end{aligned}
$$

and the velocity field is obtained from the stream function via

$$
\boldsymbol{u}_{h}=\nabla^{\perp} \psi_{h} \text {. }
$$

Clearly the velocity $\boldsymbol{u}_{h}$ satisfies the divergence free condition everywhere, and the normal velocity $\boldsymbol{u}_{h} \cdot \boldsymbol{n}$ is continuous across any element boundary. The treatment of the convection term in (1.5) is legitimate even though the tangential velocity is not continuous across the element boundaries.

Finite element approximations of the form (1.5) have been studied for the case of steady state flow. However, the computation of the vorticity and stream function are fully coupled, cf. [14]. See 77, 12] for the case of the biharmonic equation and [2, 1, 5, 18, 25] for the steady Navier-Stokes equation. The main contribution of this paper is an efficient time stepping procedure of (1.5) which will be described in Section 2. All the nice features mentioned before in the finite difference setting will be maintained for the finite element method. At the same time the method is generalized in an extremely simple and clean way for the general domains in both $2 \mathrm{D}$ and 3D, with arbitrary order of accuracy. The high order spectral element method can be easily adapted into our scheme. More complicated physics, such as 
stratified flow, gravitation and centrifugal forces, turbulence models, etc., can be incorporated into the scheme.

There is also a simple and natural generalization of the above methodology to 3D, which will be discussed in Section 3. It is well known that there is a major difference between two and three dimensions for vorticity-based numerical methods. Most apparent of all is the fact that both the vorticity and stream function become vector (instead of scalar) fields in 3D. At the same time, the stream function changes its name to vector potential. Along with this is the necessity to enforce the solenoidal conditions for the vorticity and vector potential. This turns out to be a major problem in designing efficient numerical methods in 3D based on this formulation.

In Section 4, we will present an error analysis for both 2D and 3D. A near optimal estimate of $h^{k-1 / 2}$ in $L^{2}$-norm for velocity and vorticity will be proved for the standard $k$ th order finite element method. Systemic numerical experiments of the methods proposed in this paper will be reported in [20] for the standard finite element method, in [19] for discontinuous Galerkin method.

\section{EXPLICIT TIME-STEPPING PROCEDURE}

Since finite element methods amount to centered schemes, a high order time discretization method must be used in order to avoid cell Reynolds number constraints. This is discussed in detail in [9. In practice we use the classical fourth order Runge-Kutta method, which can essentially be written as four forward Euler type steps [9]. For example, the intermediate stage $u^{* *}$ in RK4 for the ODE $u^{\prime}=f(u)$ can be formulated as

$$
u^{* *}=u^{n}+\frac{1}{2} \Delta t f\left(u^{*}\right) .
$$

Therefore, we will illustrate the time discretization of (1.5) using forward Euler.

Suppose we know the values of $\omega^{n}, \psi^{n}$ and $\boldsymbol{u}^{n}$ at $t^{n}$. We first compute an auxiliary term $\left\langle\varphi, \omega^{n+1}\right\rangle$ for any $\varphi \in X_{0, h}^{k}$ from

$$
\left\langle\varphi, \omega^{n+1}\right\rangle=\left\langle\varphi, \omega^{n}\right\rangle+\Delta t\left\langle\nabla \varphi, \omega^{n} \boldsymbol{u}^{n}\right\rangle-\nu \Delta t\left\langle\nabla \varphi, \nabla \omega^{n}\right\rangle .
$$

Using this auxiliary term, we can solve for stream function $\psi^{n+1} \in X_{0, h}^{k}$ from

$$
\left\langle\nabla \varphi, \nabla \psi^{n+1}\right\rangle=-\left\langle\varphi, \omega^{n+1}\right\rangle, \quad \forall \varphi \in X_{0, h}^{k} .
$$

From $\psi^{n+1}$, we can obtain the vorticity $\omega^{n+1}$ by inverting a mass matrix from

$$
\left\langle\varphi, \omega^{n+1}\right\rangle=\left\langle\nabla \varphi, \nabla \psi^{n+1}\right\rangle, \quad \forall \varphi \in X_{h}^{k} .
$$

The right hand side of the above equation does not have to be computed again for each test function $\varphi \in X_{0, h}^{k}$ since it is equal to the auxiliary term from (II), which has already been computed in (I). Finally we compute the velocity

$$
\boldsymbol{u}^{n+1}=\nabla^{\perp} \psi^{n+1} \text {. }
$$

We should emphasize that in the above time stepping procedure, the momentum equation (I) is completely decoupled from the kinematic equation (II). There is no iteration required between the vorticity and stream function to recover the boundary values for the vorticity. Many existing methods require this, for example [21, 2, 1, 22, 16, 14].

At first sight, this procedure seems circular since (II) and (III) seem to use the same equation, but are used to compute different things. So some comments are in order: 
1. Step (I) only computes $\left(\varphi, \omega^{n+1}\right)$ for $\varphi \in X_{0, h}^{k}$. To completely determine $\omega^{n+1}$ we need to compute $\left(\varphi, \omega^{n+1}\right)$ for all $\varphi \in X_{h}^{k}$. The computation of $\left(\varphi, \omega^{n+1}\right)$ for the degrees of freedom associated with the boundary nodes is split into two steps. First in Step (II) we compute the stream-function $\psi^{n+1}$. Fortunately, and this is very important for the success of this procedure, knowing $\left(\varphi, \omega^{n+1}\right)$ for $\varphi \in X_{0, h}^{k}$ is enough to compute $\psi^{n+1}$. This is the same reason why the explicit methods work so well in the finite difference setting. Having $\psi^{n+1}$, we then compute $\left(\varphi, \omega^{n+1}\right)$ for $\varphi \in X_{h}^{k} \backslash X_{0, h}^{k}$ using step (III). However, (III) is also valid for $\varphi \in X_{0, h}^{k}$ because of (II).

2. The equation $\left(\varphi, \omega^{n+1}\right)=\left(\nabla \varphi, \nabla \psi^{n+1}\right)$ for $\varphi \in X_{h}^{k} \backslash X_{0, h}^{k}$ can be thought of as the vorticity boundary condition. This is a natural generalization of Thom's formula.

The above procedure can be described more clearly with matrix notation. Let $\phi_{i}, i=1,2, \cdots, N_{0}$, be a basis corresponding to the interior nodes, and $\phi_{j}, j=$ $N_{0}+1, N_{0}+2, \cdots, N_{0}+N_{b}$, a basis corresponding to the boundary nodes. Denote $\omega^{n}=\left(\omega_{i}\right)$ and $\psi^{n}=\left(\psi_{i}\right)$, vectors of size $\left(N_{0}+N_{b}\right)$ and $N_{0}$, representing the vorticity and stream function

$$
\omega_{h}^{n}=\sum_{i=1}^{N_{0}+N_{b}} \omega_{i} \phi_{i}, \quad \psi_{h}^{n}=\sum_{i=1}^{N_{0}} \psi_{i} \phi_{i},
$$

respectively. For the Lagrangian finite element space, the number of nodes is equal to the dimension of the finite element space, i.e.,

$$
N_{0}=\text { dimension of } X_{0, h}^{k}, \quad N_{0}+N_{b}=\text { dimension of } X_{h}^{k} .
$$

Denote by $M=\left(\left\langle\phi_{i}, \phi_{j}\right\rangle\right)$ the standard $\left(N_{0}+N_{b}\right) \times\left(N_{0}+N_{b}\right)$ mass matrix and by $A=\left(\left\langle\nabla \phi_{i}, \nabla \phi_{j}\right\rangle\right)$ the $\left(N_{0}+N_{b}\right) \times\left(N_{0}+N_{b}\right)$ stiffness matrix, partitioned, according to the dimensions $N_{0}$ and $N_{b}$, as

$$
A=\left(\begin{array}{cc}
A_{0,0} & A_{0, b} \\
A_{b, 0} & A_{b, b}
\end{array}\right) .
$$

The auxiliary term in (I) is denoted by $\bar{\omega}^{n+1}=\left(\bar{\omega}_{i}\right)$, a vector of size $N_{0}$ and having values

$$
\bar{\omega}_{\ell}=\sum_{i=1}^{N_{0}+N_{b}}\left\langle\phi_{\ell}, \phi_{i}\right\rangle \omega_{i}, \quad \ell=1,2, \cdots, N_{0} .
$$

We denote the nonlinear term by $\mathcal{N}\left(\boldsymbol{u}^{n}, \omega^{n}\right)=\left(\mathcal{N}_{i}\right)$, a vector of size $N_{0}$ and having values

$$
\mathcal{N}_{\ell}=-\sum_{i=1}^{N_{0}+N_{b}} \sum_{j=1}^{N_{0}}\left\langle\nabla \phi_{\ell}, \phi_{i} \nabla^{\perp} \phi_{j}\right\rangle \omega_{i} \psi_{j}, \quad \ell=1,2, \cdots, N_{0} .
$$

Then the momentum equation (I) can be written as

$$
\frac{\bar{\omega}^{n+1}-\bar{\omega}^{n}}{\Delta t}+\mathcal{N}\left(\boldsymbol{u}^{n}, \omega^{n}\right)=\nu\left(A_{0,0}, A_{0, b}\right) \omega^{n} .
$$

We can use the auxiliary variable $\bar{\omega}^{n+1}$ to solve for the stream function $\psi^{n+1}$ from

$$
A_{0,0} \psi^{n+1}=\bar{\omega}^{n+1} \text {. }
$$


We can solve for $\omega^{n+1}$ by inverting the mass matrix $M$ from

$$
M \omega^{n+1}=\left(\begin{array}{c}
\bar{\omega}^{n+1} \\
A_{b, 0} \psi^{n+1}
\end{array}\right)
$$

The velocity is again given by $(2.1)$.

The above time stepping procedure is very similar to the one used in an essentially compact fourth order finite difference scheme (EC4 for short) proposed in [10. The vorticity boundary value on the left side of $\left(\mathrm{III}^{\prime}\right)$ is related to the stream function $\psi^{n+1}$ through the local kinematic relation on the right hand side of (III'). The boundary values of $\omega^{n+1}$ are obtained along with the interior values in (III') by inverting a mass matrix. This is quite general and natural and does not result in any iteration between the vorticity and stream function. The simplicity and efficiency of the above scheme is obvious. The main computation involves solving a standard Poisson equation ( $\left.\mathrm{II}^{\prime}\right)$ and inverting a standard mass matrix (III'). Standard FEM packages with a Poisson solver can easily be modified to compute the unsteady viscous incompressible flow.

Alternatively, the finite element discretization of $\left(1.4^{\prime}\right)$ results in a symmetric Galerkin form: find $\psi_{h} \in X_{0, h}^{k}$ and $\omega_{h} \in X_{h}^{k}$ such that

$$
\begin{aligned}
\left\langle\nabla \varphi, \nabla \partial_{t} \psi_{h}\right\rangle+\left\langle\nabla \varphi, \omega_{h} \boldsymbol{u}_{h}\right\rangle & =\nu\left\langle\nabla \varphi, \nabla \omega_{h}\right\rangle, & \forall \varphi \in X_{0, h}^{k}, \\
\left\langle\varphi, \omega_{h}\right\rangle & =-\left\langle\nabla \varphi, \nabla \psi_{h}\right\rangle, \quad & \forall \varphi \in X_{h}^{k} .
\end{aligned}
$$

In a matrix notation as defined before, $\left(1.5^{\prime}\right)$ becomes

$$
\begin{aligned}
A_{0,0} \partial_{t} \psi_{h}+\mathcal{N}\left(\boldsymbol{u}_{h}, \omega_{h}\right) & =\nu\left(A_{0,0}, A_{0, b}\right) \omega_{h}, \\
M \omega_{h} & =-\left(\begin{array}{c}
A_{0,0} \\
A_{b, 0}
\end{array}\right) \psi_{h} .
\end{aligned}
$$

Explicit time discretization of above equation is equivalent to the time-stepping (I)(III'). Equation (1.5') can be viewed as an ODE system for $\psi_{h}$ after eliminating $\omega_{h}$.

\section{Finite Element Method \\ IN THE 3D VORTICITY-VECTOR POTENTIAL FORMULATION}

Now we present the finite element method in the $3 \mathrm{D}$ vorticity-vector potential formulation. A corresponding finite difference version was studied in [11]. We will use the following 3D Navier-Stokes equation in the vorticity-vector potential formulation:

$$
\begin{aligned}
\partial_{t} \boldsymbol{\omega}+\nabla \times(\boldsymbol{\omega} \times \boldsymbol{u}) & =\nu \Delta \boldsymbol{\omega}, \\
\Delta \boldsymbol{\psi} & =-\boldsymbol{\omega},
\end{aligned}
$$

with six boundary conditions

$$
\begin{aligned}
\boldsymbol{n} \times \boldsymbol{\psi}=0, \quad \nabla \cdot \boldsymbol{\psi} & =0, \\
\nabla \cdot \boldsymbol{\omega}=0, \quad \boldsymbol{n} \times(\nabla \times \boldsymbol{\psi}) & =\boldsymbol{n} \times \boldsymbol{u}_{b} .
\end{aligned}
$$

The velocity is given by

$$
\boldsymbol{u}=\nabla \times \boldsymbol{\psi} .
$$

It is easy to verify that (3.1)-(3.3) imply

$$
\nabla \cdot \boldsymbol{\omega}=0, \quad \nabla \cdot \boldsymbol{u}=0, \quad \nabla \cdot \boldsymbol{\psi}=0 .
$$

As a consequence of (3.4), (3.1)-(3.3) are equivalent to the Navier-Stokes equation in primitive variables. See [23, Theorem 3.1] for a more detailed discussion. For 
simplicity we assume $\boldsymbol{u}_{b}=0$. General inhomogeneous boundary conditions can be treated similarly. The difficulty in designing an efficient numerical method for (3.1)(3.3) arises from the need to enforce the solenoidal conditions (3.4) numerically. We will use a div-curl type stiffness matrix (see (3.6) below) in our finite element approximation to overcome this difficulty. Define the space $Y=\left(H^{1}\right)^{3}$ and the subspace

$$
Y_{\tau}=\{\boldsymbol{\psi} \in Y: \boldsymbol{n} \times \boldsymbol{\psi}=0 \quad \text { on } \Gamma\} .
$$

It was proved in [8] that the $\|\cdot\|_{H^{1}}$ norm in $Y_{\tau}$ is equivalent to the div-curl norm

$$
\lfloor\phi\rceil \equiv \sqrt{[\phi, \phi]}, \quad[\phi, \psi] \equiv\langle\nabla \cdot \phi, \nabla \cdot \boldsymbol{\psi}\rangle+\langle\nabla \times \phi, \nabla \times \boldsymbol{\psi}\rangle,
$$

and that the following Poincaré inequality holds:

$$
\|\phi\| \leq C\lfloor\phi\rceil, \quad \forall \phi \in Y_{\tau} .
$$

Indeed, it was shown in [8] that for any $\phi, \psi$ in $Y_{\tau}$,

$$
\langle\nabla \phi, \nabla \boldsymbol{\psi}\rangle=[\boldsymbol{\phi}, \boldsymbol{\psi}]+\int_{\Gamma} \frac{2}{R} \phi \cdot \boldsymbol{\psi} d \gamma
$$

where $R$ is the mean radius of the curvature of $\Gamma$. $R>0$ when $\Omega$ is convex. A variational form for (3.1) and (3.2) is given as follows. Find $\boldsymbol{\omega} \in Y$ and $\boldsymbol{\psi} \in Y_{\tau}$ such that

$$
\begin{aligned}
\left\langle\boldsymbol{\phi}, \partial_{t} \boldsymbol{\omega}\right\rangle+\langle\nabla \times \boldsymbol{\phi}, \boldsymbol{\omega} \times \boldsymbol{u}\rangle & =-\nu[\boldsymbol{\phi}, \boldsymbol{\omega}], \quad \forall \boldsymbol{\phi} \in Y_{\tau}, \\
{[\boldsymbol{\phi}, \boldsymbol{\psi}] } & =\langle\boldsymbol{\phi}, \boldsymbol{\omega}\rangle, \quad \forall \boldsymbol{\phi} \in Y,
\end{aligned}
$$

or alternatively in a more symmetric way, find $\boldsymbol{\psi} \in Y_{\tau}$ and $\boldsymbol{\omega} \in Y$ such that

$$
\begin{aligned}
{\left[\boldsymbol{\phi}, \partial_{t} \boldsymbol{\psi}\right]+\langle\nabla \times \boldsymbol{\phi}, \boldsymbol{\omega} \times \boldsymbol{u}\rangle } & =-\nu[\boldsymbol{\phi}, \boldsymbol{\omega}], \quad \forall \boldsymbol{\phi} \in Y_{\tau}, \\
\langle\boldsymbol{\phi}, \boldsymbol{\omega}\rangle & =[\boldsymbol{\phi}, \boldsymbol{\psi}], \quad \forall \boldsymbol{\phi} \in Y .
\end{aligned}
$$

Again, the velocity is given by (3.3). It is easy to verify that (3.9) is equivalent to (3.1)-(3.2). See [3] for discussions on the variational formulation in multiconnected domains.

Let $Y_{h}^{k}=\left(X_{h}^{k}\right)^{3}$ be the standard continuous finite element space with degree $k$ and

$$
Y_{\tau, h}^{k}=\left\{\boldsymbol{\psi} \in Y_{h}^{k}: \boldsymbol{n} \times \boldsymbol{\psi}=0 \quad \text { on } \Gamma\right\} .
$$

In general, the boundary condition in (3.10) is understood to be valid for all the boundary nodes. The finite element approximation of (3.9) is given as follows. Find $\boldsymbol{\omega}_{h} \in Y_{h}^{k}$ and $\boldsymbol{\psi}_{h} \in Y_{\tau, h}^{k}$ such that

$$
\begin{aligned}
\left\langle\boldsymbol{\phi}, \partial_{t} \boldsymbol{\omega}_{h}\right\rangle+\left\langle\nabla \times \boldsymbol{\phi}, \boldsymbol{\omega}_{h} \times \boldsymbol{u}_{h}\right\rangle & =-\nu\left[\boldsymbol{\phi}, \boldsymbol{\omega}_{h}\right], \quad \forall \boldsymbol{\phi} \in Y_{\tau, h}^{k}, \\
{\left[\boldsymbol{\phi}, \boldsymbol{\psi}_{h}\right] } & =\left\langle\boldsymbol{\phi}, \boldsymbol{\omega}_{h}\right\rangle, \quad \forall \boldsymbol{\phi} \in Y_{h}^{k},
\end{aligned}
$$

or, written in a symmetric Galerkin form, find $\boldsymbol{\psi}_{h} \in Y_{\tau, h}^{k}$ and $\boldsymbol{\omega}_{h} \in Y_{h}^{k}$ such that

$$
\begin{aligned}
{\left[\boldsymbol{\phi}, \partial_{t} \boldsymbol{\psi}_{h}\right]+\left\langle\nabla \times \boldsymbol{\phi}, \boldsymbol{\omega}_{h} \times \boldsymbol{u}_{h}\right\rangle } & =-\nu\left[\boldsymbol{\phi}, \boldsymbol{\omega}_{h}\right], \quad \forall \boldsymbol{\phi} \in Y_{\tau, h}^{k}, \\
\left\langle\boldsymbol{\phi}, \boldsymbol{\omega}_{h}\right\rangle & =\left[\boldsymbol{\phi}, \boldsymbol{\psi}_{h}\right], \quad \forall \boldsymbol{\phi} \in Y_{h}^{k},
\end{aligned}
$$

and the velocity is given by

$$
\boldsymbol{u}_{h}=\nabla \times \boldsymbol{\psi}_{h} .
$$


Explicit time-stepping procedure. As in 2D, we now present an efficient time stepping scheme for (3.11)-(3.12). Again, we use forward Euler as an illustration.

Suppose we know the values of $\boldsymbol{\omega}^{n}, \boldsymbol{\psi}^{n}$ and $\boldsymbol{u}^{n}$ at $t^{n}$. We first compute an auxiliary term $\left\langle\phi, \boldsymbol{\omega}^{n+1}\right\rangle$ for any $\phi \in Y_{\tau, h}^{k}$ from

$$
\left\langle\boldsymbol{\phi}, \boldsymbol{\omega}^{n+1}\right\rangle=\left\langle\boldsymbol{\phi}, \boldsymbol{\omega}^{n}\right\rangle-\Delta t\left\langle\nabla \times \boldsymbol{\phi}, \boldsymbol{\omega}^{n} \times \boldsymbol{u}^{n}\right\rangle-\nu \Delta t\left[\boldsymbol{\phi}, \boldsymbol{\omega}^{n}\right] .
$$

Using this auxiliary term we can solve for the vector potential $\psi^{n+1} \in Y_{\tau, h}^{K}$ from

$$
\left[\phi, \boldsymbol{\psi}^{n+1}\right]=\left\langle\phi, \boldsymbol{\omega}^{n+1}\right\rangle, \quad \forall \phi \in Y_{\tau, h}^{k} .
$$

From $\boldsymbol{\psi}^{n+1}$, we can obtain the vorticity $\boldsymbol{\omega}^{n+1}$ by inverting a mass matrix from

$$
\left\langle\phi, \boldsymbol{\omega}^{n+1}\right\rangle=\left[\phi, \psi^{n+1}\right], \quad \forall \phi \in Y_{h}^{k} .
$$

The right hand side of the above equation does not have to be computed again for each test function $\phi \in Y_{\tau, h}^{k}$ since it is equal to the auxiliary term from (B), which has already been computed in (A). Finally we compute the velocity

$$
\boldsymbol{u}^{n+1}=\nabla \times \boldsymbol{\psi}^{n+1} .
$$

Remark 1. When the boundary of the domain consists of flat surfaces, i.e., a polygon, then the mean radius of curvature $R=\infty$. From (3.8), we know that for any $\phi, \psi \in Y_{\tau}$,

$$
\langle\nabla \phi, \nabla \boldsymbol{\psi}\rangle=[\phi, \boldsymbol{\psi}] .
$$

The stiffness matrix in (B) can be replaced by the standard stiffness matrix for the vector Poisson equation. In the case when the boundary surfaces are parallel to the coordinate planes, the three components of the vector potential decouple, and the boundary conditions in (B) become homogeneous Dirichlet boundary conditions for the tangential components of the vector potential, and Neumann boundary condition for the normal component. This special geometric property has been explored before in a finite difference approximation [11].

Designing simple and efficient finite difference schemes for 3D in vorticity-vector potential formulations have been attacked in [11 with the same strategy: treat the viscous term explicitly and use a local vorticity boundary formula. A second order and fourth order compact difference scheme on a nonstaggered grid was proposed in [11], and a local vorticity boundary condition, analogous to Thom's formula in $2 \mathrm{D}$, is derived.

\section{Stability AND ERRor eStimates FOR THE SEMI-Discrete CASE}

The stability analysis and error estimates for the finite element approx -imations (1.5)-(1.6) in 2D and (3.11)-(3.12) in 3D are rather standard. For completeness, we give a detailed account here. For the stability analysis, we have the following conservation of energy:

Theorem 1. Let $\boldsymbol{u}_{h}$ and $\omega_{h}$ be the solution of the finite approximation (1.5)-(1.6) for the $2 D$ Navier Stokes equation (1.1)-(1.3). Then we have

$$
\left\|\boldsymbol{u}_{h}(\cdot, t)\right\|_{L^{2}}^{2}+2 \nu \int_{0}^{t}\left\|\omega_{h}(\cdot, s)\right\|_{L^{2}}^{2} d s=\left\|\boldsymbol{u}_{h}(\cdot, 0)\right\|_{L^{2}}^{2} .
$$


Let $\boldsymbol{u}_{h}, \boldsymbol{\omega}_{h}$ and $\boldsymbol{\psi}_{h}$ be the solution of the finite element approximation (3.11)-(3.12) for 3D Navier Stokes equation (4.1)-(4.3). Then we have

$$
\begin{aligned}
& \left\|\boldsymbol{u}_{h}(\cdot, t)\right\|_{L^{2}}^{2}+\left\|\nabla \cdot \boldsymbol{\psi}_{h}(\cdot, t)\right\|_{L^{2}}^{2}+2 \nu \int_{0}^{t}\left\|\boldsymbol{\omega}_{h}(\cdot, s)\right\|_{L^{2}}^{2} d s \\
& \quad=\left\|\boldsymbol{u}_{h}(\cdot, 0)\right\|_{L^{2}}^{2}+\left\|\nabla \cdot \boldsymbol{\psi}_{h}(\cdot, 0)\right\|_{L^{2}}^{2} .
\end{aligned}
$$

Proof. We first prove (4.1). Take $\varphi=\psi_{h}$ in the first equation of (1.5) to obtain

$$
\left\langle\psi_{h}, \partial_{t} \omega_{h}\right\rangle-\left\langle\nabla \psi_{h}, \omega_{h} \boldsymbol{u}_{h}\right\rangle+\nu\left\langle\nabla \psi_{h}, \nabla \omega_{h}\right\rangle=0 .
$$

The second term is zero since $\boldsymbol{u}_{h} \cdot \nabla \psi_{h}=0$. Taking $\varphi=\omega_{h}$ in the second equation of (1.5), we have

$$
\left\langle\nabla \psi_{h}, \nabla \omega_{h}\right\rangle+\left\langle\omega_{h}, \omega_{h}\right\rangle=0 .
$$

Take the time derivative of the second equation of (1.5), and replace $\varphi$ by $\psi_{h}$ to obtain

$$
\left\langle\psi_{h}, \partial_{t} \omega_{h}\right\rangle=-\left\langle\nabla \psi_{h}, \partial_{t} \nabla \psi_{h}\right\rangle=-\frac{d}{d t} \frac{1}{2}\left\|\nabla \psi_{h}\right\|^{2}=-\frac{d}{d t} \frac{1}{2}\left\|\boldsymbol{u}_{h}\right\|^{2} .
$$

We obtain from (4.3) and (4.4) the conservation of energy

$$
\frac{d}{d t}\left\|\boldsymbol{u}_{h}\right\|^{2}+2 \nu\left\|\omega_{h}\right\|^{2}=0 \text {. }
$$

Integration in time gives (4.1). The proof for the 3D problem is similar. We take $\phi=\boldsymbol{\psi}_{h}$ in the first equation of (3.11) to obtain

$$
\left\langle\boldsymbol{\psi}_{h}, \partial_{t} \boldsymbol{\omega}_{h}\right\rangle+\left\langle\nabla \times \boldsymbol{\psi}_{h}, \boldsymbol{\omega}_{h} \times \boldsymbol{u}_{h}\right\rangle+\nu\left[\boldsymbol{\psi}_{h}, \boldsymbol{\omega}_{h}\right]=0 .
$$

The second term is zero since $\left(\nabla \times \boldsymbol{\psi}_{h}\right) \cdot \boldsymbol{\omega}_{h} \times \boldsymbol{u}_{h}=0$. Taking $\boldsymbol{\phi}=\boldsymbol{\omega}_{h}$ in the second equation of (3.11), we have

$$
\left[\boldsymbol{\psi}_{h}, \boldsymbol{\omega}_{h}\right]=\left\langle\boldsymbol{\omega}_{h}, \boldsymbol{\omega}_{h}\right\rangle .
$$

As in (4.6), we have from the second equation of (3.11) that

$$
\left\langle\partial_{t} \boldsymbol{\omega}_{h}, \boldsymbol{\psi}_{h}\right\rangle=\frac{d}{d t} \frac{1}{2}\left\lfloor\boldsymbol{\psi}_{h}\right\rceil^{2} .
$$

We obtain from (4.7) and (4.8) the conservation of energy

$$
\frac{d}{d t}\left\lfloor\boldsymbol{\psi}_{h}\right\rceil^{2}+2 \nu\left\|\boldsymbol{\omega}_{h}\right\|^{2}=0 .
$$

Integrating in time and using the fact that $\boldsymbol{u}_{h}=\nabla \times \boldsymbol{\psi}$, we obtain (4.2). This completes the proof of Theorem 1.

Ladyzhenskaya-Babuska-Brezzi (LBB) condition. The finite element approximations (1.5) and (3.11) can be viewed as a mixed approximation of the NavierStokes equations (1.1)-(1.2) and (3.1)-(3.2), respectively. The LBB condition plays an important role in the error analysis of mixed type problems [4, 13, 16]. We should emphasize that in the fully discrete scheme (1.7)-(1.10), the momentum equation (1.7) is completely decoupled from the kinematic equation (1.8). Nevertheless the LBB condition is still important when choosing the discrete spatial spaces. The same is true for the $3 \mathrm{D}$ problem.

The LBB condition for (1.5) is

$$
\inf _{\psi_{h} \in X_{0, h}^{k}} \sup _{\varphi_{h} \in X_{h}^{k}} \frac{\left\langle\nabla \varphi_{h}, \nabla \psi_{h}\right\rangle}{\left\|\varphi_{h}\right\|\left\|\nabla \psi_{h}\right\|} \geq C>0,
$$


and the LBB condition for (3.11) is

$$
\inf _{\boldsymbol{\psi}_{h} \in Y_{\tau, h}^{k}} \sup _{\boldsymbol{\phi}_{h} \in Y_{h}^{k}} \frac{\left[\boldsymbol{\phi}_{h}, \boldsymbol{\psi}_{h}\right]}{\left\|\boldsymbol{\phi}_{h}\right\|\left\lfloor\boldsymbol{\psi}_{h}\right\rceil} \geq C>0 .
$$

Using the Poincaré inequality for $\psi_{h}$ we can obtain (4.10) directly from

$$
\sup _{\varphi_{h} \in X_{h}^{k}} \frac{\left\langle\nabla \varphi_{h}, \nabla \psi_{h}\right\rangle}{\left\|\varphi_{h}\right\|} \geq \frac{\left\langle\nabla \psi_{h}, \nabla \psi_{h}\right\rangle}{\left\|\psi_{h}\right\|} \geq C\left\|\nabla \psi_{h}\right\| .
$$

Using the Poincaré inequality (3.7), estimation of (4.11) is similar. Directly using the LBB condition will give an $h^{k-1}$ estimate for $\omega$ and $\boldsymbol{u}$ for the biharmonic equation or the steady Stokes equation, cf. [4]. A more careful analysis using an $L^{\infty}$-estimate or duality argument will give an $h^{k-1 / 2}$ accuracy cf. [24, 12]. The time dependence and the nonlinear term will contribute some more complications. We will give a detailed account of the error estimates for the full time dependent NSE in both 2D and 3D below.

Some estimates for projection operators. We first define two projection operators for the $2 \mathrm{D}$ finite element spaces. $P$ is the standard $L^{2}$ projection into the space $X_{h}^{k}$

$$
\langle\varphi, \omega-P \omega\rangle=0, \quad \forall \varphi \in X_{h}^{k}
$$

and $\Pi$ is the standard projection into $X_{0, h}^{k}$

$$
\langle\nabla \varphi, \nabla(\psi-\Pi \psi)\rangle=0, \quad \forall \varphi \in X_{0, h}^{k} .
$$

We have the standard estimates for $P$ and $\Pi[6]$

$$
\|\nabla(\boldsymbol{\phi}-P \boldsymbol{\phi})\|+\|\nabla(\boldsymbol{\phi}-\Pi \boldsymbol{\phi})\| \leq C h^{k}\|\boldsymbol{\phi}\|_{H^{k+1}},
$$

and a maximum estimate 6

$$
\|\psi-\Pi \psi\|_{1, \infty} \leq C h^{k}|\ln h|^{\alpha}\|\psi\|_{W^{k+1, \infty}},
$$

where $\alpha=1$ if $k=1$; otherwise $\alpha=0$.

Similarly, we define the two projection operators for the 3D finite element spaces. $P$ is the standard $L^{2}$ projection into the space $Y_{h}^{k}$

$$
\langle\phi, \boldsymbol{\omega}-P \boldsymbol{\omega}\rangle=0, \quad \forall \boldsymbol{\phi} \in Y_{h}^{k} ;
$$

and we have the standard estimates [6]

$$
\|\nabla(\phi-P \phi)\| \leq C h^{k}\|\phi\|_{H^{k+1}},
$$

and $\Pi$ is the projection into $Y_{\tau, h}^{k}$

$$
[\boldsymbol{\phi}, \boldsymbol{\psi}-\Pi \boldsymbol{\psi}]=0, \quad \forall \boldsymbol{\phi} \in Y_{\tau, h}^{k} .
$$

It was proved in 8] that

$$
\lfloor\phi-\Pi \phi\rceil \leq C h^{k}\|\phi\|_{H^{k+1}}
$$

for any $\phi \in Y \cap\left(H^{k+1}\right)^{3}$ and $\nabla \cdot \phi=0$. 
Error estimate for 2D case. We now proceed to estimate the error for the finite element approximation (1.5) for the Navier-Stokes equation (1.4). Let $\omega$ and $\psi$ be the exact solution of (1.4). We denote the error functions by

$$
\varepsilon=\omega-\omega_{h}, \quad \delta=\psi-\psi_{h} .
$$

Since both the numerical solution and the exact solution satisfy (1.5), one has after subtracting one from another,

$$
\begin{aligned}
\left\langle\varphi, \partial_{t} \varepsilon\right\rangle-\left\langle\nabla \varphi,\left(\omega \boldsymbol{u}-\omega_{h} \boldsymbol{u}_{h}\right)\right\rangle+\nu\langle\nabla \varphi, \nabla \varepsilon\rangle & =0 \quad \forall \varphi \in X_{0, h}^{k}, \\
\langle\nabla \varphi, \nabla \delta\rangle+\langle\varphi, \varepsilon\rangle & =0 \quad \forall \varphi \in X_{h}^{k} .
\end{aligned}
$$

Following the standard strategy for estimating the error in a finite element approximation for the evolution type equation [17], we will first estimate the following projections of the error terms in (4.20):

$$
\varepsilon_{h}=P \varepsilon=P \omega-\omega_{h}, \quad \delta_{h}=\Pi \delta=\Pi \psi-\psi_{h} .
$$

Using (4.12) we obtain from (4.21)

$$
\begin{aligned}
\left\langle\varphi, \partial_{t} \varepsilon_{h}\right\rangle-\left\langle\nabla \varphi,\left(\omega \boldsymbol{u}-\omega_{h} \boldsymbol{u}_{h}\right)\right\rangle+\nu\langle\nabla \varphi, \nabla \varepsilon\rangle & =0 \quad \forall \varphi \in X_{0, h}^{k}, \\
\langle\nabla \varphi, \nabla \delta\rangle+\left\langle\varphi, \varepsilon_{h}\right\rangle & =0 \quad \forall \varphi \in X_{h}^{k} .
\end{aligned}
$$

Take $\varphi=\delta_{h}$ in the first equation of (4.23). The first term can be estimated in a manner similar to that used to estimate (4.6)

$$
\left\langle\delta_{h}, \partial_{t} \varepsilon_{h}\right\rangle=-\frac{d}{d t} \frac{1}{2}\left\|\nabla \delta_{h}\right\|^{2} .
$$

The second term becomes

$$
\begin{aligned}
\left\langle\nabla \delta_{h},\left(\omega \boldsymbol{u}-\omega_{h} \boldsymbol{u}_{h}\right)\right\rangle & =\left\langle\nabla \delta_{h}, \omega\left(\boldsymbol{u}-\boldsymbol{u}_{h}\right)\right\rangle+\left\langle\nabla \delta_{h}, \varepsilon \boldsymbol{u}_{h}\right\rangle \\
& =\left\langle\nabla \delta_{h}, \omega \nabla^{\perp}\left(\delta-\delta_{h}\right)\right\rangle+\left\langle\nabla \delta_{h}, \varepsilon \boldsymbol{u}_{h}\right\rangle \\
& =\left\langle\nabla \delta_{h}, \omega \nabla^{\perp}(\psi-\Pi \psi)\right\rangle+\left\langle\nabla \delta_{h}, \varepsilon \boldsymbol{u}_{h}\right\rangle .
\end{aligned}
$$

In the second equality above we have used the fact that $\delta_{h} \cdot \nabla^{\perp} \delta_{h}=0$. Assuming for the moment that

$$
\left\|\boldsymbol{u}_{h}\right\|_{\infty} \leq \tilde{C}
$$

the second order term can be estimated as

$$
\left\langle\nabla \delta_{h},\left(\omega \boldsymbol{u}-\omega_{h} \boldsymbol{u}_{h}\right)\right\rangle \leq C_{1}\left\|\nabla \delta_{h}\right\|(\|\nabla(\psi-\Pi \psi)\|+\|\varepsilon\|),
$$

where

$$
C_{1}=C\left(\tilde{C}+\|\omega\|_{L^{\infty}}\right) .
$$

Together with (4.23) and (4.24) we have

$$
\frac{d}{d t}\left\|\nabla \delta_{h}\right\|^{2} \leq 2 \nu\left\langle\nabla \delta_{h}, \nabla \varepsilon\right\rangle+C_{1}\left\|\nabla \delta_{h}\right\|(\|\nabla(\psi-\Pi \psi)\|+\|\varepsilon\|) .
$$

To estimate the first term on the right hand side of (4.27), we take $\varphi=\varepsilon_{h}$ in the second equation of (4.23). Direct computation leads to

$$
\begin{aligned}
\left\langle\nabla \delta_{h}, \nabla \varepsilon\right\rangle & =-\left\langle\varepsilon_{h}, \varepsilon_{h}\right\rangle+\left\langle\nabla \delta_{h}, \nabla\left(\varepsilon-\varepsilon_{h}\right)\right\rangle-\left\langle\nabla \varepsilon_{h}, \nabla\left(\delta-\delta_{h}\right)\right\rangle \\
& =-\left\langle\varepsilon_{h}, \varepsilon_{h}\right\rangle+\left\langle\nabla \delta_{h}, \nabla(\omega-P \omega)\right\rangle-\left\langle\nabla \varepsilon_{h}, \nabla(\psi-\Pi \psi)\right\rangle .
\end{aligned}
$$


Plugging back into (4.27), we obtain

$$
\begin{gathered}
\frac{d}{d t}\left\|\nabla \delta_{h}\right\|^{2}+2 \nu\left\|\varepsilon_{h}\right\|^{2} \leq C_{1}\left\|\nabla \delta_{h}\right\|\left(\|\nabla(\psi-\Pi \psi)\|+\|\nabla(\omega-P \omega)\|+\left\|\varepsilon_{h}\right\|\right) \\
\quad-2 \nu\left\langle\nabla \varepsilon_{h}, \nabla(\psi-\Pi \psi)\right\rangle \\
\leq C_{1}\left\|\nabla \delta_{h}\right\|\left(\left\|\varepsilon_{h}\right\|+h^{k}\left(\|\psi\|_{H^{k+1}}+\|\omega\|_{H^{k+1}}\right)\right) \\
-2 \nu\left\langle\nabla \varepsilon_{h}, \nabla(\psi-\Pi \psi)\right\rangle .
\end{gathered}
$$

In the last inequality, we have used the estimate (4.14). To estimate the last term on the right hand side of (4.28), we denote by $\varepsilon_{h, 0} \in X_{o, h}^{k}$ the interior components of $\varepsilon_{h}$ and by $\varepsilon_{h, b}$ the boundary components of $\varepsilon_{h}$, i.e.,

$$
\varepsilon_{h}=\varepsilon_{h, 0}+\varepsilon_{h, b} .
$$

Using the projection (4.13), we have

$$
\left\langle\nabla \varepsilon_{h}, \nabla(\psi-\Pi \psi)\right\rangle=\left\langle\nabla \varepsilon_{h, b}, \nabla(\psi-\Pi \psi)\right\rangle .
$$

Direct estimation gives

$$
\begin{aligned}
\left\langle\nabla \varepsilon_{h, b}, \nabla(\psi-\Pi \psi)\right\rangle & \leq C h\|\psi-\Pi \psi\|_{1, \infty} \sum^{\prime}\left|\varepsilon_{h, b}\right| \\
& \leq C h^{k+1}\|\psi\|_{W^{k+1, \infty}} \sum^{\prime}\left|\varepsilon_{h, b}\right|
\end{aligned}
$$

with a $|\ln h|$ factor on the right hand side of (4.29) if $k=1$. Here the summation $\sum^{\prime}$ is over the boundary nodes. The boundary nodes on the right hand side of (4.29) can be directly estimated as follows:

$$
\sum^{\prime}\left|\varepsilon_{h, b}\right| \leq C h^{-1 / 2} \sqrt{\sum^{\prime}\left|\varepsilon_{h, b}\right|^{2}} \leq C h^{-3 / 2}\left\|\varepsilon_{h, b}\right\| \leq C h^{-3 / 2}\left\|\varepsilon_{h}\right\| .
$$

Together with (4.28) we have

$$
\begin{aligned}
\frac{d}{d t}\left\|\nabla \delta_{h}\right\|^{2}+2 \nu\left\|\varepsilon_{h}\right\|^{2} \leq & C_{1} h^{k}\left\|\nabla \delta_{h}\right\|\left(\|\psi\|_{H^{k+1}}+\|\omega\|_{H^{k+1}}\right) \\
& +\left\|\varepsilon_{h}\right\|\left(C_{1}\left\|\nabla \delta_{h}\right\|+C h^{k-1 / 2}\|\psi\|_{W^{k+1, \infty}}\right) .
\end{aligned}
$$

Using Hölder's inequality, we have

$$
\frac{d}{d t}\left\|\nabla \delta_{h}\right\|^{2}+\nu\left\|\varepsilon_{h}\right\|^{2} \leq \frac{C_{1}^{2}}{\nu}\left\|\nabla \delta_{h}\right\|^{2}+\frac{C h^{2 k-1}}{\nu}\left(\|\psi\|_{W^{k+1, \infty}}^{2}+\|\omega\|_{H^{k+1}}^{2}\right) .
$$

The Grownwall inequality gives

$$
\left\|\nabla \delta_{h}(\cdot, t)\right\|_{L^{\infty}\left((0, T] ; L^{2}\right)}+\left\|\varepsilon_{h}(\cdot, t)\right\|_{L^{2}\left((0, T] ; L^{2}\right)} \leq C_{2} h^{k-1 / 2}
$$

where

$$
C_{2}=\left(\|\psi\|_{L^{\infty}\left((0, T] ; W^{k+1, \infty}\right)}+\|\omega\|_{L^{\infty}\left((0, T] ; H^{k+1}\right)}\right) e^{C_{1}^{2} T / \nu} / C_{1} .
$$

Therefore

$$
\begin{aligned}
\left\|\boldsymbol{u}-\boldsymbol{u}_{h}\right\| & =\left\|\nabla^{\perp}\left(\psi-\psi_{h}\right)\right\|=\left\|\nabla\left(\psi-\psi_{h}\right)\right\| \\
& \leq\|\nabla(\psi-\Pi \psi)\|+\left\|\nabla \delta_{h}\right\| \leq C_{2} h^{k-1 / 2} .
\end{aligned}
$$

Using an inverse inequality, we have

$$
\left\|\boldsymbol{u}-\boldsymbol{u}_{h}\right\|_{\infty} \leq C_{2} h^{k-3 / 2} .
$$


This justifies the $a$ priori assumption (4.25) when $k \geq 2$ by taking

$$
\tilde{C}=\|\boldsymbol{u}\|_{\infty}+C_{2} h^{k-3 / 2} .
$$

Therefore we have proved the the following theorem.

Theorem 2. Let $(\psi, \omega, \boldsymbol{u})$ be an exact solution to the Navier-Stokes equation (1.4) and $\left(\psi_{h}, \omega_{h}, \boldsymbol{u}_{h}\right)$ be the numerical solution of the finite element approximation (1.5)(1.6) with standard $k$ th order finite element space $X_{h}^{k}, k \geq 2$. Then we have

$$
\begin{aligned}
&\left\|\boldsymbol{u}-\boldsymbol{u}_{h}\right\|_{L^{\infty}\left((0, T] ; L^{2}\right)}+\left\|\omega-\omega_{h}\right\|_{L^{2}\left((0, T] ; L^{2}\right)} \\
& \leq C h^{k-1 / 2}\left(\|\psi\|_{L^{\infty}\left((0, T] ; W^{k+1, \infty}\right)}+\|\omega\|_{L^{\infty}\left((0, T] ; H^{k+1}\right)}\right) \\
& \times \exp \frac{C T\left(\|\omega\|_{\infty}^{2}+\|\boldsymbol{u}\|_{\infty}^{2}\right)}{\nu},
\end{aligned}
$$

where $C$ is a constant that does not depend on $h$ or the solution.

Error estimate for $3 \mathrm{D}$ case. As in 2D, we let $\boldsymbol{\omega}$ and $\boldsymbol{\psi}$ be the exact solution to (3.9) and define the error functions by

$$
\boldsymbol{\epsilon}=\boldsymbol{\omega}-\boldsymbol{\omega}_{h}, \quad \boldsymbol{\delta}=\boldsymbol{\psi}-\boldsymbol{\psi}_{h},
$$

and their projections by

$$
\boldsymbol{\epsilon}_{h}=P \boldsymbol{\epsilon}=P \boldsymbol{\omega}-\boldsymbol{\omega}_{h}, \quad \boldsymbol{\delta}_{h}=\Pi \boldsymbol{\delta}=\Pi \boldsymbol{\psi}-\boldsymbol{\psi}_{h} .
$$

Since both the numerical solution and the exact solution satisfy (3.11), we have

$$
\begin{aligned}
\left\langle\boldsymbol{\phi}, \partial_{t} \boldsymbol{\epsilon}_{h}\right\rangle+\left\langle\nabla \times \boldsymbol{\phi},\left(\boldsymbol{\omega} \times \boldsymbol{u}-\boldsymbol{\omega}_{h} \times \boldsymbol{u}_{h}\right)\right\rangle+\nu[\boldsymbol{\phi}, \boldsymbol{\epsilon}] & =0 \quad \forall \boldsymbol{\phi} \in Y_{\tau, h}^{k}, \\
{\left[\boldsymbol{\phi}_{h}, \boldsymbol{\delta}\right]+\left\langle\boldsymbol{\phi}_{h}, \boldsymbol{\epsilon}_{h}\right\rangle } & =0 \quad \forall \boldsymbol{\phi} \in Y_{h}^{k} .
\end{aligned}
$$

Above we have used the property of the projection (4.16).

Take $\boldsymbol{\phi}=\boldsymbol{\delta}_{h}$ in the first equation of (4.36). The first term becomes

$$
\frac{d}{d t} \frac{1}{2}\left\lfloor\boldsymbol{\delta}_{h}\right\rceil^{2}=\left\langle\boldsymbol{\delta}_{h}, \partial_{t} \boldsymbol{\omega}_{h}\right\rangle,
$$

and the second term becomes

$$
\begin{aligned}
\left\langle\nabla \times \boldsymbol{\delta}_{h},\left(\boldsymbol{\omega} \times \boldsymbol{u}-\boldsymbol{\omega}_{h} \times \boldsymbol{u}_{h}\right)\right\rangle & =\left\langle\nabla \times \boldsymbol{\delta}_{h}, \boldsymbol{\omega} \times\left(\boldsymbol{u}-\boldsymbol{u}_{h}\right)\right\rangle+\left\langle\nabla \times \boldsymbol{\delta}_{h}, \boldsymbol{\epsilon} \times \boldsymbol{u}_{h}\right\rangle \\
& =\left\langle\nabla \times \boldsymbol{\delta}_{h}, \boldsymbol{\omega} \times \nabla \times\left(\boldsymbol{\delta}-\boldsymbol{\delta}_{h}\right)\right\rangle+\left\langle\nabla \times \boldsymbol{\delta}_{h}, \boldsymbol{\epsilon} \times \boldsymbol{u}_{h}\right\rangle .
\end{aligned}
$$

Assuming for the moment that

$$
\left\|\boldsymbol{u}_{h}\right\|_{\infty} \leq C
$$

we have

$$
\left\langle\nabla \times \boldsymbol{\delta}_{h},\left(\boldsymbol{\omega} \times \boldsymbol{u}-\boldsymbol{\omega}_{h} \times \boldsymbol{u}_{h}\right)\right\rangle \leq C\left\|\nabla \times \boldsymbol{\delta}_{h}\right\|(\|\nabla \times(\boldsymbol{\psi}-\Pi \boldsymbol{\psi})\|+\|\boldsymbol{\epsilon}\|) .
$$

From (4.36), (4.37) and (4.39) we have

$$
\frac{d}{d t}\left\lfloor\boldsymbol{\delta}_{h}\right\rceil^{2} \leq 2 \nu\left[\boldsymbol{\delta}_{h}, \boldsymbol{\epsilon}\right]+C\left\lfloor\boldsymbol{\delta}_{h}\right\rceil(\lfloor\boldsymbol{\psi}-\Pi \boldsymbol{\psi}\rceil+\|\boldsymbol{\epsilon}\|) .
$$

To estimate the first term on the right hand side of (4.40), we take $\boldsymbol{\phi}=\boldsymbol{\delta}_{h}$ in the second equation of (4.36). Direct computation leads to

$$
\left[\boldsymbol{\delta}_{h}, \boldsymbol{\epsilon}\right]=\left\langle\boldsymbol{\epsilon}_{h}, \boldsymbol{\epsilon}_{h}\right\rangle+\left[\boldsymbol{\delta}_{h},\left(\boldsymbol{\omega}-\Pi \boldsymbol{\omega}_{h}\right)\right]-\left[\boldsymbol{\epsilon}_{h},(\boldsymbol{\psi}-\Pi \boldsymbol{\psi})\right] .
$$

The last term can be estimated by the inverse inequality,

$$
\left[\epsilon_{h},(\boldsymbol{\psi}-\Pi \psi)\right] \leq \frac{C}{h}\left\|\epsilon_{h}\right\|\lfloor\boldsymbol{\psi}-\Pi \psi\rceil .
$$


Plugging (4.42) and (4.41) back to (4.40), we have

$$
\begin{gathered}
\frac{d}{d t}\left\lfloor\boldsymbol{\delta}_{h}\right\rceil^{2}+2 \nu\left\|\boldsymbol{\epsilon}_{h}\right\|^{2} \leq C\left\lfloor\boldsymbol{\delta}_{h}\right\rceil\left(\lfloor\boldsymbol{\psi}-\Pi \boldsymbol{\psi}\rceil+\|\nabla(\boldsymbol{\omega}-P \boldsymbol{\omega})\|+\left\|\boldsymbol{\epsilon}_{h}\right\|\right) \\
+\nu \frac{C}{h}\left\|\boldsymbol{\epsilon}_{h}\right\|\lfloor\boldsymbol{\psi}-\Pi \boldsymbol{\psi}\rceil .
\end{gathered}
$$

Using the estimates (4.17) and (4.19) for the first two terms on the right hand side of (4.43) we have

$$
\frac{d}{d t}\left\lfloor\boldsymbol{\delta}_{h}\right\rceil^{2}+\nu\left\|\boldsymbol{\epsilon}_{h}\right\|^{2} \leq C\left\lfloor\boldsymbol{\delta}_{h}\right\rceil^{2}+C h^{2 k-2} .
$$

This gives

$$
\left\lfloor\boldsymbol{\delta}_{h}\right\rceil \leq C h^{k-1} .
$$

Using an inverse inequality, we are able to justify the a priori assumption (4.38) when $k \geq 2$. We now summarize the above arguments in the following theorem.

Theorem 3. Let $(\boldsymbol{\psi}, \boldsymbol{\omega}, \boldsymbol{u})$ be an exact solution to the Navier-Stokes equation (3.9) and $\left(\boldsymbol{\psi}_{h}, \boldsymbol{\omega}_{h}, \boldsymbol{u}_{h}\right)$ be the numerical solution of the finite element approximation (3.11)-(3.12) with standard $k$ th order finite element space $X_{h}^{k}, k \geq 3$. Then we have

$$
\begin{aligned}
\| \boldsymbol{u}- & \boldsymbol{u}_{h}\left\|_{L^{\infty}\left((0, T] ; L^{2}\right)}+\right\| \boldsymbol{\omega}-\boldsymbol{\omega}_{h}\left\|_{L^{2}\left((0, T] ; L^{2}\right)}+\right\| \nabla \cdot \boldsymbol{\psi}_{h} \|_{L^{\infty}\left(0, T, L^{2}\right)} \\
& \leq C h^{k-1}\left(\|\psi\|_{L^{\infty}\left((0, T] ; H^{k+1}\right)}+\|\omega\|_{L^{\infty}\left((0, T] ; H^{k+1}\right)}\right) \\
& \times \exp \frac{C T\left(\|\omega\|_{\infty}^{2}+\|\boldsymbol{u}\|_{\infty}^{2}\right)}{\nu}
\end{aligned}
$$

Here $C$ is a constant that does not depend on $h$ or the solution.

We remark that an $h^{1 / 2}$ order shaper estimate can be improved in (4.46), provided the following maximum norm estimate for the projection of $\Pi$ in (4.18) is true:

$$
\lfloor\boldsymbol{\psi}-\Pi \boldsymbol{\psi}\rceil_{\infty} \leq C h^{k}|\ln h|^{\alpha}\|\boldsymbol{\psi}\|_{W^{k+1, \infty}}
$$

where

$$
\lfloor\boldsymbol{\psi}\rceil_{\infty}=\|\nabla \cdot \boldsymbol{\psi}\|_{\infty}+\|\nabla \times \boldsymbol{\psi}\|_{\infty}
$$

$\alpha=1$ if $k=1$; otherwise $\alpha=0$.

\section{Concluding Remarks}

We presented a very simple, efficient and accurate finite element method for the unsteady incompressible Navier-Stokes equation (NSE) in vorticity-vector potential (stream function in 2D) formulation. The standard continuous finite element space with $k$ th degree polynomials is used for both the vorticity and vector potential. A near optimal error estimate of $h^{k-1 / 2}$ is obtained for both velocity and vorticity.

The efficiency of the method results from the explicit time stepping procedure that we described in Section 1 for 2D and in Section 2 for 3D. The viscous term is treated explicitly and the boundary vorticity is determined by a local explicit formula from the vector potential (stream function) via the kinematic relation at no additional cost. The resulting momentum equation is completely decoupled from the kinematic equation. There is no iteration between the vorticity and the vector potential (stream function). At each time step or Runge-Kutta stage the main 
computation involves solving a standard Poisson equation and inverting a standard mass matrix. In the 3D case, the stiffness matrix is of div-curl form and is used to achieve the solenoidal conditions for the vorticity and vector-potential. This is a key difficulty in designing efficient numerical methods for NSE for 3D. In the case when the domain is a polygon, the div-curl form stiffness matrix is reduced to the standard stiffness matrix for the vector Poisson equation. Standard FEM packages with a Poisson solver can be easily modified to compute the unsteady viscous incompressible flow.

A finite difference version of the above scheme has been studied in detail and successfully used in many applications [9, 10, 11]. The time stepping follows exactly the same steps in the essentially compact fourth difference scheme [10. A variational formulation and finite element approximation give a very general, natural, and clean way to implement the above methodology. It overcomes the difficulty of finding an accurate local vorticity formula for a curved boundary, especially in the 3D case.

\section{REFERENCES}

1. E. Barragy and G.F. Carey, Stream function vorticity solution using high-p element-byelement techniques, Commun. in Applied Numer. Methods, 9 (1993) 387-395.

2. K.E. Barrett, A variational principle for the stream function-vorticity formulation of the Navier-Stokes equations incorporating no-slip conditions, J. Comput. Phys. 26 (1978) 153161. MR 57:8429

3. A. Bendali, J.M. Dominguez and S. Gallic, A variational approach for the vector potential formulation of the Stokes and Navier-Stokes problems in three dimensional domains, J. Math. Anal. Appl. 107 (1985) 537-560. MR 86k:35121

4. F. Brezzi and M. Fortin, Mixed and hybrid finite element methods, Springer-Verlag, New York, 1991. MR 92d:65187

5. A. Campion-Renson and M. J. Crochet, On the stream function-vorticity finite element solutions of Navier-Stokes equations, Int. J. Num. Meth. Engng. 12 (1978) 1809-1818.

6. P. Ciarlet, The Finite Element Method for Elliptic Problems, North-Holland, Amsterdam, 1978. MR 58:25001

7. P. Ciarlet and P. Raviart, A mixed finite element method for the biharmonic equation, in "Symposium on Mathematical Aspects of Finite Elements in Partial Differential Equations", C. de Boor, ed, Academic Press, New York, (1974) 125-143. MR 58:31907

8. F. El Dabaghi and O. Pironneau, Stream vector in three dimensional aerodynamics, Numer. Math. 48 (1986) 561-589. MR 87h:65193

9. Weinan E and J.-G. Liu, Vorticity boundary condition and related issues for finite difference schemes, J. Comput. Phys., 124 (1996), 368-382. MR 97a:76088

10. Weinan E and J.-G. Liu, Essentially compact schemes for unsteady viscous incompressible flows, J. Comput. Phys., 126 (1996), 122-138. MR 97e:76050

11. Weinan E and J.-G. Liu, Finite difference methods in vorticity-vector potential formulation on $3 D$ non-staggered grids, J. Comput. Phys., 138 (1997) 57-82.

12. R.S. Falk and J.E. Osborn, Error estimates for mixed methods, R.A.I.R.O. Anal. Numer. 14 (1980) 249-277. MR 82j:65076

13. V. Girault and P.A. Raviart, Finite Element Methods for Navier-Stokes Equations, Theory and Algorithms, Springer-Verlag, Berlin, (1986). MR 88b:65129

14. P.M. Gresho, Incompressible fluid dynamics: some fundamental formulation issues, Ann. Rev. Fluid Mech. 23 (1991), 413-453. MR 92e:76017

15. P.M. Gresho, Some interesting issues in incompressible fluid dynamics, both in the continuum and in numerical simulation, Advances in Applied Mechanics, 28 (1992), 45-140. MR 93e:76056

16. M. Gunzburger, Finite element methods for viscous incompressible flows, Academic Press, Boston, 1989 MR 91d:76053

17. C. Johnson and V. Thomée, Error estimates for some mixed finite element methods for parabolic type problems, R.A.I.R.O. Anal. Numer. 15 (1981) 41. MR 83c:65239 
18. M. Ikegawa, A new finite element technique for the analysis of steady viscous flow problems, Int. J. Num. Meth. Engng. 14 (1979) 103-113.

19. J.-G. Liu and C.-W. Shu, A high order discontinuous Galerkin method for incompressible flows, J. Comput. Phys., (to appear).

20. J.-G. Liu and J. Xu, An efficient finite element method for viscous incompressible flows, in preparation.

21. S.A. Orszag and M. Israeli, Numerical simulation of viscous incompressible flows, Ann. Rev. Fluid Mech., 6 (1974), 281-318.

22. O.A. Pironneau, Finite Element Methods for Fluids, John Wiley and Sons (1989) MR 90j:76016

23. L. Quartapelle, Numerical Solution of the Incompressible Navier-Stokes Equations, Birkhäuser, Berlin, (1993). MR 95i:76060

24. R. Schulz, A mixed method for fourth order problems using linear elements, R.A.I.R.O. Anal. Numer. 12 (1978) 85-90.

25. W.N.R. Stevens, Finite element, stream function-vorticity solution of steady laminar natural convection, Int. J. Num. Meth. Fluids 2 (1982) 349-366.

Institute for Physical Science and Technology and Department of Mathematics, University of Maryland, College Park, MD 20742

E-mail address: jliu@math.umd.edu

Courant Institute of Mathematical Sciences, New York, NY 10012

E-mail address: weinan@cims.nyu.edu 